\title{
WHEN DO OPTIMA CONVERGE TO OPTIMUM?*
}

\section{Abstract}

In this paper we consider the problem of the convergence of optima in approximated problems to the optimum of limiting problem. We recall the concept of $\Gamma$-convergence, which is crucial in optimisation problems. We show its importance and provide a list of theorems describing its properties. Using described tools we derive the conditions under which an approximation of the parameters of the Ramsey model with vanishing absolute error of approximation gives the explicite formula for the time-varying consumption per capita, which leads to "almost"-maximisation of the CRRA utility functional.

Keywords: Gamma-convergence, Ramsey model, convergence of optima, stability of optimal paths.

JEL Classification: C02, C62.

\section{Introduction}

There are several types of convergence of sequences of functions. The usual and well-known types are pointwise and uniform convergence. In this paper we present the basis of the theory of $\Gamma$-convergence of sequence of functions. This kind of convergence can be expressed also in terms of Kuratowski convergence of epigraphs of those functions (limit of sequence of closed sets).

$\Gamma$-convergence is a crucial concept in optimisation problems. It is worth emphasising that it is not obvious that if we have a sequence of functions

Marta Kornafel, Cracow University of Economics, Faculty of Finance and Law, Department of Mathematics, Rakowicka 27, 31-510 Kraków, Poland, e-mail: marta.kornafel@uek.krakow.pl

* This publication presents the results of a research project financed from a subsidy for the maintenance of research potential, granted to the Faculty of Finance and Law at the Cracow University of Economics. 
- or functionals (Attouch 1984), possibly profit, cost or utility functionals each of which possesses an extremum at some point, then the limit of functions has an extremum in the limiting point of the optima sequence. We start our analysis in the second section with an elementary example that shows the opposite effect for a pointwise limit of sequence of functions. In the next part of the paper we formulate a formal definition of the $\Gamma$-limit and discuss the theorems linking it to other types of limits of sequence of functions.

The paper closes with an analysis of the standard Ramsey model, in which we consider a disturbance of coefficients. We provide an answer to the natural question: in what circumstances does the optimal consumption path in a "disturbed" problem approximate the optimal consumption path from the "ideal" model and under what conditions does blanking the coefficients disturbance lead to the non-disturbed optimum.

\section{The Idea of I-convergence - an Example}

$\Gamma$-convergence is one of the most important tools of optimisation theory, which assures the convergence of both minima and minimisers to, respectively, the minimum and minimiser of the limiting functional. The problem of convergence of optima was first considered by E. De Giorgi (1984), who introduced and developed the concept of $\Gamma$-convergence. In parallel, mathematicians studied the problem of convergence of sets, also in optimisation problems. There was the question of how to extend the Hausdorff metric, defined for nonempty and compact (therefore, in $\mathbb{R}^{n}$ : closed and bounded) sets, to the case of closed sets. Kazimierz Kuratowski was the first to prepare a fundamental description of this convergence and showed the equivalence between $\Gamma$-convergence of functionals and the Kuratowski convergence of their epigraphs (Kuratowski 1961).

We are now going to present a brief outline of both approaches. First, we give a definition of epigraph and - using this intuitive geometric approach discuss an example illustrating the fundamental role of $\Gamma$-convergence.

Definition 1. Let function $f: X \rightarrow \mathbb{R}$ be given, where $X$ is a topological space. The epigraph of function $f$ is the set:

$$
\text { epi }(f):=\{(x, v) \in X \times \mathbb{R}: v \geq f(x)\} .
$$

Geometrically, epigraph of function is the part of Cartesian product $X \times \mathbb{R}$ above the graph of $f$. For a lower semicontinuous function $f$ its graph is a closed subset of the Cartesian product $X \times \mathbb{R}$. 
Example 1. The epigraph of function $f(x)=x^{4}-3 x^{3}+x-4$ is presented in Figure 1.

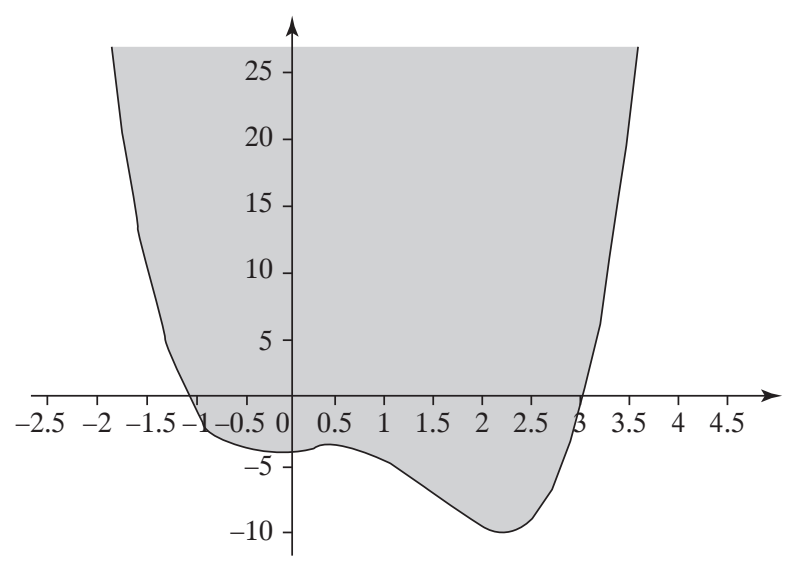

Fig. 1. The Epigraph of Function $f$

Source: author's own elaboration.

Example 2. Consider the following sequence of functions $(n \in \mathbb{N})$ :

$$
f_{n}(x)=\left\{\begin{aligned}
1, & -2 \leq x<-\frac{2}{n} \\
(1-2 n) x+\frac{2-3 n}{n}, & -\frac{2}{n}<x \leq-\frac{1}{n} \\
(2 n-1) x+1, & -\frac{1}{n}<x \leq 0 \\
-x+1, & 0<x \leq 1 .
\end{aligned}\right.
$$

Their graphs (for exemplary values of parameter $n$ ) are presented in Figure 2.

It is easy to observe that each function $f_{n}$ possess the minimum at point $x_{n}=-\frac{1}{n}$, and the minimal value is $y_{n}=f_{n}\left(x_{n}\right)=-1+\frac{1}{n}$. Therefore, calculating trivial limits one obtains that $\bar{x}=\lim _{n \rightarrow+\infty} x_{n}=0$ and $\bar{y}=\lim _{n \rightarrow+\infty} y_{n}=-1$, being respectively the limits of minimisers and of minima. 
Consider now the limit of sequence $\left(f_{n}\right)$, in two approaches. The pointwise limit is obviously the function:

$$
f_{p}(x)=\left\{\begin{aligned}
1, & -1 \leq x<0 \\
-x+1, & 0 \leq x \leq 1
\end{aligned}\right.
$$

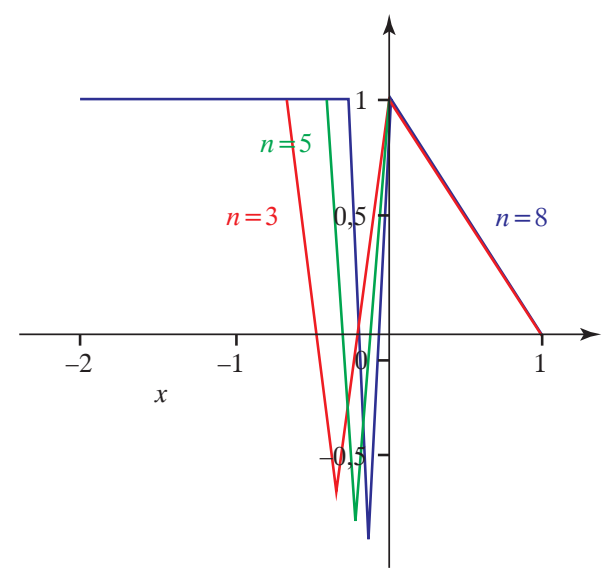

Fig. 2. Graphs of Functions $f_{n}$ Source: author's own elaboration.

However, if we consider the epigraphs of functions $f_{n}$, it is easy to observe that they converge to the set in Figure 3.

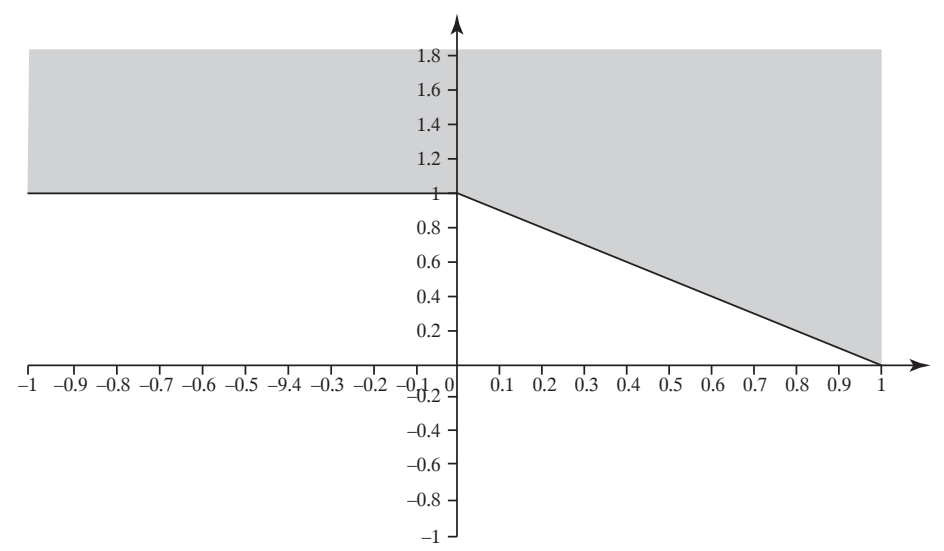

Fig. 3. The Kuratowski Limit of Sequence $\left(e p i\left(f_{n}\right)\right)_{n \in \mathbb{N}}$ Source: author's own elaboration. 
Therefore, the function whose epigraph is represented by this limiting set is:

$$
f_{e p i}(x)=\left\{\begin{array}{rl}
1, & x \in[-1,0), \\
-1 & x=0, \\
-x+1, & x \in(0,1] .
\end{array}\right.
$$

Since $f_{\text {epi }}(0) \neq f_{p}(0)$, we have that $f_{e p i} \neq f_{p}$.

Let us have a look at the variational properties of both limit functions, $f_{p}$ and $f_{e p i}$. The minimal value of $f_{p}$ is attained at $x_{p}=1$ and $y_{p}=\min _{x \in[-1,1]} f_{p}(x)=0$, while the minimiser and minimal value for $f_{e p i}$ are $x_{e p i}=0$ and $y_{e p i}=\min _{x \in[-1,1]} f_{e p i}(x)=-1$. Notice that the last two coincide with the limits $\bar{x}$ and $\bar{y}$. Therefore, function $f_{e p i}$ has good variational properties: it is the limit of our sequence $\left(f_{n}\right)$ - more precisely: the $\Gamma$-limit of this sequence; the limit of minimisers $\left(x_{n}\right)$ is a minimiser of the limit $f_{e p i}$ and finally the limit of minima $\left(y_{n}\right)$ is in fact the minimum of the $\operatorname{limit} f_{e p i}$.

The observed phenomenon is not incidental. In fact, it was the reason to define $\Gamma$-convergence. Those properties are crucial in optimisation problems. They assure the stability of optima and justify the usage of numerical methods, which - by their nature - deal with approximate problems and face the issue of propagation of computational errors.

While studying the last example, the natural question arises: how can the $\Gamma$-limit of sequence of functions be formally defined and how is it related to the pointwise and uniform limit of sequence of functions (which are not sufficient in those kinds of problems)? Also, when discussing example 1, we used the intuitive concept of the epigraphical limit of the sequence $\left(f_{n}\right)$ and only mentioned its relation to the $\Gamma$-limit. In the next section, all those concepts are formalised.

\section{3. r-limit and Links to Other Types of Limits}

In this section, we formulate the necessary definitions and theorems on epigraphical convergence. They are cited from G. Dal Maso (1993).

In what follows, we are going to denote by $X$ a topological space ${ }^{1}$ and $f_{n}: X \rightarrow \overline{\mathbb{R}}$ (where $\overline{\mathbb{R}}=\mathbb{R} \cup\{ \pm \infty\}$ is the extended real line) a sequence of

${ }^{1}$ In the definitions and theorems, we consider $X$ to be a topological space, metric space or topological vector space. These are mathematical structures, allowing usage of different operations on the elements of set $X$, like measuring the distance or operating on vectors, respectively. For 
functions defined in it, while $N(x)$ denotes the family of open neighbourhoods of the point $x$.

Definition 2 (Dal Maso 1993, p. 38). The lower and upper $\Gamma$-limits of the sequence $\left(f_{n}\right)$ are defined as follows:

$$
\begin{gathered}
f_{\text {low }-\Gamma}:=\Gamma-\liminf _{n \rightarrow \infty} f_{n}(x):=\sup _{U \in N(x)} \liminf _{n \rightarrow \infty} \inf _{y \in U} f_{n}(y), \\
f_{\text {upp }-\Gamma}:=\Gamma-\limsup _{n \rightarrow \infty} f_{n}(x):=\sup _{U \in N(x)} \limsup _{n \rightarrow \infty} \inf _{y \in U} f_{n}(y) .
\end{gathered}
$$

If $f_{\text {low }-\Gamma}=f_{\text {upp }-\Gamma}:=f_{\Gamma}$, then sequence $\left(f_{n}\right)$ is said to be $\Gamma$-convergent to $f_{\Gamma}$ and function $f_{\Gamma}$ is $\Gamma$-limit of it.

We define now the lower and upper Kuratowski limits of the family of sets (net), $\left(E_{n}\right) \subset X$, when $n \rightarrow \infty$.

Definition 3 (Dal Maso 1993, p. 41). The lower and upper Kuratowski limits of a sequence of sets are, respectively:

$$
\begin{aligned}
& x \in K-\liminf _{n \rightarrow \infty} E_{n} \Leftrightarrow \forall U \in N(x) \quad \exists k \in \mathbb{N} \forall h \geq k: U \cap E_{h} \neq \varnothing \\
& x \in K-\limsup _{n \rightarrow \infty} E_{n} \Leftrightarrow \forall U \in N(x) \quad \forall k \in \mathbb{N} \exists h \geq k: U \cap E_{h} \neq \varnothing .
\end{aligned}
$$

Clearly, $K-\lim \inf _{n \rightarrow \infty} E_{n} \subset K-\lim \sup _{n \rightarrow \infty} E_{n}$. If the converse inclusion holds as well, we denote the resulting set by $E:=K-\lim _{n \rightarrow \infty} E_{n}$ and call it the Kuratowski limit of $\left(E_{n}\right)$ when $n \rightarrow \infty$. Therefore, $\left(E_{n}\right)$ converges to some set $E$ as $n \rightarrow \infty$, iff:

$$
K-\limsup _{n \rightarrow \infty} E_{n} \subset E \subset K-\liminf _{n \rightarrow \infty} E_{n} .
$$

The next theorem establishes the relation between $\Gamma$-convergence of sequence of functions and the Kuratowski convergence of their epigraphs.

Theorem 1 (Dal Maso 1993, p. 44). Let $f_{\text {low }-\Gamma}$ and $f_{\text {upp }-\Gamma}$ be respectively the lower and upper limits of a sequence of functions $\left(f_{n}\right)$. Then:

$$
\begin{aligned}
& \operatorname{epi}\left(f_{\text {low }-\Gamma}\right)=K-\limsup _{n \rightarrow \infty} \operatorname{epi}\left(f_{n}\right), \\
& \operatorname{epi}\left(f_{\text {upp }-\Gamma}\right)=K-\liminf _{n \rightarrow \infty} \operatorname{epi}\left(f_{n}\right) .
\end{aligned}
$$

Therefore, $\left(f_{n}\right) \Gamma$-converges to $f_{\Gamma}$ if and only if epi $\left(f_{\Gamma}\right)=K-\lim _{n \rightarrow \infty} e p i\left(f_{n}\right)$. 
We are ready to formulate the theorem which describes the fundamental role of $\Gamma$-convergence in optimisation theory. This is particular case of the more general theorem 7.12 in Dal Maso (1993, p. 73).

For a function $f: X \rightarrow \overline{\mathbb{R}}$ denote by $M(f)$ the set of its minimisers (possibly empty), i.e.:

$$
M(f):=\left\{x \in X: f(x)=\inf _{y \in X} f(y)\right\} .
$$

Theorem 2. Assume that sequence $\left(f_{n}\right)$ is $\Gamma$-convergent to $f_{\Gamma}$. Then:

a) $K-\lim \inf _{n \rightarrow \infty} M\left(f_{n}\right) \subset M\left(f_{\Gamma}\right)$,

i.e. any limit of a sequence of minima $y_{n}$ is a minimiser of $f_{\Gamma}$;

b) if $K-\lim _{n \rightarrow \infty} M\left(f_{n}\right) \neq \varnothing$, then $M\left(f_{\Gamma}\right) \neq \varnothing$ and $\min _{x \in X} f_{\Gamma}(x)=$ $=\lim _{n \rightarrow \infty}\left(\inf _{x \in X} f_{n}(x)\right)$,

i.e. if there exists a limit of a sequence of minima $y_{n}$, then function $f_{\Gamma}$ has at least one minimiser (this limit itself, maybe also some other) and the minimum of $f_{\Gamma}$ is approximated by minima $y_{n}$;

c) if $f_{\Gamma}$ is a proper function (not identically $+\infty$ ), then:

$$
M\left(f_{\Gamma}\right) \subset K-\limsup _{n \rightarrow \infty} M\left(f_{n}\right),
$$

i.e. if $f_{\Gamma}$ has at least one finite value, then any minimiser of this function is the limit of a sequence of minimisers $y_{n}$.

As we observed in example 1 , both pointwise and $\Gamma$-limits of the considered sequence of functions existed. In general, these are independent concepts and it may happen that one of those limits exists while the other does not. However, there are some situations when they both do exist and coincide. Below we cite the theorems from Dal Maso (1993) describing most important cases, usual in economic modelling.

The subscript " $\mathrm{p}$ " is related to pointwise limit.

\section{Theorem 3}

a) $f_{\text {low }-\Gamma} \leq f_{\text {low }-p}$ and $f_{\text {upp }-\Gamma} \leq f_{\text {upp }-p}$. In particular, if both the $\Gamma$-limit $f_{\Gamma}$ and pointwise limit $f_{p}$ exist, then $f_{\Gamma} \leq f_{p}$.

b) If each function $f_{n}$ is continuous and sequence $\left(f_{n}\right)$ converges uniformly ${ }^{2}$ to a function $f$, then $f$ is continuous and $f=f_{\Gamma}$.

c) If $\left(f_{n}\right)$ is an increasing sequence of continuous functions, then $f_{\Gamma}=\sup _{n \in \mathbb{N}} f_{n}$.

${ }^{2}$ Having $(X, d)$ a metric space with distance function $d$, sequence of functions $f_{n}: X \rightarrow \overline{\mathbb{R}}$ is uniformly convergent to function $f: X \rightarrow \overline{\mathbb{R}}$ iff $\lim _{n \rightarrow \infty} \sup _{x \in X} d\left(f_{n}(x), f(x)\right)=0$. 
d) Let $X$ be normed vector space. If $\left(f_{n}\right)$ is a sequence of equi-bounded ${ }^{3}$ in a neighbourhood of a point $x \in X$ and convex functions, then - provided the sequence $\left(f_{n}\right)$ is convergent $-f_{\Gamma}=f_{p}$.

The next theorem determines when the properties of convexity and homogeneity are inherited by $\Gamma$-limits.

Theorem 4. Let $X$ be a topological vector space over the real numbers. Then:

a) if $\left(f_{n}\right)$ is a sequence of convex functions, then $f_{u p p-\Gamma}$ is a convex function. In particular, for $\Gamma$-convergent sequence the $\Gamma$-limit $f_{\Gamma}$ is convex;

b) if $\left(f_{n}\right)$ is a sequence of positively homogeneous of degree $p$ functions ${ }^{4}$, then both $f_{\text {low }-\Gamma}$ and $f_{\text {upp }-\Gamma}$ are positively homogeneous of degree $p$. In particular, if the sequence $\left(f_{n}\right) \Gamma$-converges to $f_{\Gamma}$, then $f_{\Gamma}$ is positively homogeneous of degree $p$.

Notice that due to the obvious facts:

1) if function $f$ attains minimum at a point $x_{0}$, then function $-f$ attains maximum at this point;

2 ) if function $f$ is increasing in a set $A$, then function $-f$ is decreasing in this set;

3 ) if function $f$ is convex in a set $X$, then function $-f$ is concave in the same set;

4) $\quad \lim \sup _{n \rightarrow \infty}\left(-f_{n}(x)\right)=-\lim _{\inf _{n \rightarrow \infty}} f_{n}(x)$ and $\liminf _{n \rightarrow \infty}\left(-f_{n}(x)\right)=$ $=-\lim \sup _{n \rightarrow \infty} f_{n}(x)$;

all the presented theorems have their corresponding formulations for functions possessing symmetric properties.

\section{Household's Utility Optimisation}

We consider now the household optimisation problem of the Ramsey model (Barro \& Sala-i-Martin 2004), in which the parameters are given with some approximation. In what follows, all the introduced functions are smooth.

The households are assumed to be identical, so they are characterised by the same preferences, the wage rate $\omega$, the rate of returns $r$ (for simplicity

\footnotetext{
${ }^{3}$ A family (or sequence) of functions $\left(f_{n}\right), f_{n}: X \rightarrow \mathbb{R}$, is equi-bounded iff there exists a constant $M>0$, which bounds any function, i.e. for any $x \in X:\left|f_{n}(x)\right|<M$.

${ }^{4}$ Having $X$ a vector topological space, function $f: X \rightarrow \overline{\mathbb{R}}$ is positively homogeneous of degree $p$ iff for any $t>0$ and any $x \in X$ it holds that $f(t x)=t^{p} f(x)$.
} 
assumed to be constant, may be understood as the average) and the same assets per person. The population grows at the rate $n>0$, so $L(t)=L(0) \cdot e^{n t}$. In further calculations, the initial size of population $L(0)$ is normalised to 1 . $C(t)$ denotes the total consumption at time $t$ and $c(t):=\frac{C(t)}{L(t)}$ consumption per capita. We consider CRRA utility, i.e.:

$$
u(c)=\frac{c^{1-\theta}-1}{1-\theta}, \quad \theta \in(0,1)
$$

so it satisfies the usual monotonicity and concavity assumptions and meets Inada conditions. Therefore, the households face the problem of choosing such a consumption path $c(t)$ to maximise the intertemporal utility functional (with discount rate $\rho>n$ ):

$$
U[c]=\int_{0}^{\infty} u(c(t)) e^{-(n-\rho) t} d t,
$$

taking into account the budget constraints and $c(t) \geq 0$. The budget constraints are expressed by the dynamics of a household's assets per person $a(t)$ :

$$
\frac{d a}{d t}=(r-n) a(t)+\omega(t)-c(t) .
$$

Ruling out the chain-letters possibilities we do require that the present value of assets is asymptotically nonnegative:

$$
\lim _{t \rightarrow \infty} a(t) \cdot e^{-(r-n)} \geq 0 .
$$

Thanks to transversality conditions and the Pontryagin Maximum Principle we can derive the optimal consumption path:

$$
c^{*}(t)=c(0) \exp \left(\frac{1}{\theta}(r-\rho) t\right) .
$$

For the derivation of the constraints and a detailed solution of the model, see R. J. Barro and X. Sala-i-Martin (2004, pp. 88-93).

Consider now the Ramsey model with "disturbed" parameters. The rates $r, n, \rho$ and parameter $\theta$ are given with some approximation, which may depend on measurement rules. Denote those approximate values by $r_{\varepsilon}$, $n_{\varepsilon}, \rho_{\varepsilon}$ and $\theta_{\varepsilon}$, respectively. Assume that for any $\varepsilon, \rho_{\varepsilon}>n_{\varepsilon}$, and $\theta_{\varepsilon} \in(0,1)$. Increasing the accuracy of measurement, the approximate values $b_{\varepsilon}$ tend to actual value $b$ for $b \in\{r, n, \rho, \theta\}$. Having those approximate values $b_{\varepsilon}$ 
the household's maximisation problem is now to maximise the functional $U_{\varepsilon}[c]=\int_{0}^{\infty} u_{\varepsilon}(c(t)) e^{-\left(n_{\varepsilon}-\rho_{\varepsilon}\right) t} d t$, where $u_{\varepsilon}(c)=\frac{c^{1-\theta_{\varepsilon}}-1}{1-\theta_{\varepsilon}}$ subject to the analogous constraints to (3) and (4). Repeating the reasoning for each $\varepsilon>0$, we obtain the optimal solution: the function $c_{\varepsilon}^{*}(t)=c(0) \exp \left(\frac{1}{\theta_{\varepsilon}}\left(r_{\varepsilon}-\rho_{\varepsilon}\right) t\right)$. The natural questions now are whether or not $c_{\varepsilon}^{*}$ converges to $c^{*}$ and $U_{\varepsilon}\left[c_{\varepsilon}^{*}\right]$ converges to $U\left[c^{*}\right]$, i.e. whether or not the optimal path $c_{\varepsilon}^{*}$ approximates the actual optimal path $c^{*}$ and $U_{\varepsilon}\left[c_{\varepsilon}^{*}\right] \approx U\left[c^{*}\right]$. To give the answer, we prove the following theorem:

Theorem 5. If $\lim _{\varepsilon \rightarrow 0} r_{\varepsilon}=r, \lim _{\varepsilon \rightarrow 0} n_{\varepsilon}=n, \lim _{\varepsilon \rightarrow 0} \rho_{\varepsilon}=\rho$ and $\lim _{\varepsilon \rightarrow 0} \theta_{e}=\theta$, then $U_{\varepsilon}$ converges to $U$ uniformly.

Proof. We will show first that the uniform limit of $\left(u_{\varepsilon}\right)$ is function $u$. Estimating the distance between $u_{\varepsilon}$ and $u$, we get:

$$
\begin{gathered}
\left|u_{\varepsilon}(c)-u(c)\right|=\left|\frac{c^{1-\theta_{\varepsilon}}-1}{1-\theta_{\varepsilon}}-\frac{c^{1-\theta}-1}{1-\theta}\right|= \\
=\left|\frac{\left(c^{1-\theta_{\varepsilon}}-c^{1-\theta}\right)+\left(\theta_{\varepsilon} \cdot c^{1-\theta}-\theta \cdot c^{1-\theta_{\varepsilon}}\right)+\left(\theta-\theta_{\varepsilon}\right)}{\left(1-\theta_{\varepsilon}\right)(1-\theta)}\right| \leq \\
\leq \frac{\left|c^{1-\theta_{\varepsilon}}-c^{1-\theta}\right|+\left|\theta_{\varepsilon} \cdot c^{1-\theta} \pm \theta_{\varepsilon} \cdot c^{1-\theta_{\varepsilon}}-\theta \cdot c^{1-\theta_{\varepsilon}}\right|+\left|\theta-\theta_{\varepsilon}\right|}{\left(1-\theta_{\varepsilon}\right)(1-\theta)} \leq \\
\leq \frac{\left(1+\theta_{\varepsilon}\right)\left|c^{1-\theta_{\varepsilon}}-c^{1-\theta}\right|+\left(c^{1-\theta_{\varepsilon}}+1\right)\left|\theta-\theta_{\varepsilon}\right|}{\left(1-\theta_{\varepsilon}\right)(1-\theta)} .
\end{gathered}
$$

Notice that if $\theta_{\varepsilon} \rightarrow \theta$, then both terms in the numerator tend to zero, so $u_{\varepsilon} \rightrightarrows u$. We are ready to prove the uniform convergence of operators $U_{\varepsilon}$ to $U$ :

$$
\begin{gathered}
\left|U_{\varepsilon}[c]-U[c]\right|=\left|\int_{0}^{\infty} u_{\varepsilon}(c(t)) e^{-\left(n_{\varepsilon}-\rho_{\varepsilon}\right) t} d t-\int_{0}^{\infty} u(c(t)) e^{-(n-\rho) t} d t\right| \leq \\
\leq \int_{0}^{\infty}\left|u_{\varepsilon}(c(t)) e^{-\left(n_{\varepsilon}-\rho_{\varepsilon}\right) t} \pm u(c(t)) e^{-\left(n_{\varepsilon}-\rho_{\varepsilon}\right) t}-u(c(t)) e^{-(n-\rho) t}\right| d t \leq \\
\leq \int_{0}^{\infty}\left[\left|u_{\varepsilon}(c(t))-u(c(t))\right| \cdot e^{-\left(\left(n_{\varepsilon}-n\right)-\left(\rho_{\varepsilon}-\rho\right)\right) t}+u(c(t)) \cdot e^{-\left(\left(n_{\varepsilon}-n\right)-\left(\rho_{\varepsilon}-\rho\right)\right) t}-1\right] \cdot e^{-(n-\rho) t} d t .
\end{gathered}
$$


Due to the uniform convergence of $\left(u_{\varepsilon}\right)$ and convergence of sequences $\left(n_{\varepsilon}\right)$ and $\left(\rho_{\varepsilon}\right)$, the integrand tends to zero function, so $U_{\varepsilon} \rightrightarrows U$, which proves the theorem.

Theorem 6. The functional $U$ is the $\Gamma$-limit of the sequence $U_{\varepsilon}$ when $\varepsilon \rightarrow 0$. Theorem 6 is the immediate consequence of theorems 5 and $3 \mathrm{~b}$.

Corollary. If parameters $r, n, \rho$ and $\theta$ are given with an approximation whose absolute error tends to zero, then the corresponding consumption path approximates the optimal "theoretical" consumption path and the obtained value of utility is close to the actual maximum.

\section{Conclusions and Further Research}

The aim of the study reported in this paper is two-fold. Firstly, we want to emphasise the role of $\Gamma$-convergence in optimisation problems, and in particular in economic modelling. Secondly, we show that using an approximation of the parameters of the Ramsey model with vanishing absolute error of approximation it is possible to give the explicite formula for the time-varying consumption per capita, which leads to "almost"-maximisation of the CRRA utility functional.

In the author's opinion it would be interesting to consider a similar problem of convergence of optimal path in the more general Ramsey model, where the rates are functions of time. Then we would enquire what kind of time-dependence of rate of returns, wages, discount rate or preference parameter still allows for the maximum of the utility functional and what kind approximation of them gives an approximation of optimal paths.

\section{Bibliography}

Attouch, H. (1984) Variational Convergence for Functions and Operators. London: Pitman. Barro, R. J. and Sala-i-Martin, X. (2004) Economic Growth. Second edition. The MIT Press.

Dal Maso, G. (1993) An Introduction to Г-Convergence. Boston, MA: Birkhäuser, https:// doi.org/10.1007/978-1-4612-0327-8.

De Giorgi, E. (1984) G-operators and $\Gamma$-convergence. Proceedings of the International Congress of Mathematicians, Warsaw 1983. Amsterdam: North Holland, pp. 1175-91. Kuratowski, K. (1961) Topologie I et II. Mathematical monograph. Second edition. PWN. 


\section{Abstract}

\section{Kiedy optima są zbieżne do optimum?}

W artykule podjęto zagadnienie zbieżności ciągu optimów zagadnień przybliżonych do optimum zagadnienia granicznego. Oparto się na $\Gamma$-zbieżności - kluczowym pojęciu w teorii optymalizacji. Wykazano istotność jej stosowania i podano twierdzenia opisujące jej najważniejsze własności. Wykorzystując wprowadzone narzędzia, wyprowadzono warunki, przy których przybliżenie parametrów modelu Ramseya (przy wygaszanym błędzie bezwzględnym aproksymacji) pozwala na podanie explicite przepisu na zmienną w czasie ścieżkę konsumpcji per capita, które prowadzi do bliskich maksimum wartości funkcjonału użyteczności typu CRRA.

Słowa kluczowe: Gamma-zbieżność, model Ramseya, zbieżność optimów, stabilność ścieżek optymalnych. 